\title{
ON THE DENSITY CHARACTER OF CLOSED SUBGROUPS
}

\author{
JOHN GINSBURG, M. RAJAGOPALAN AND VICTOR SAKS
}

\begin{abstract}
Answering a question posed by W. W. Comfort, G. L. Itzkowitz, and K. A. Ross, it is shown that for every infinite cardinal number $\alpha$ there are a Hausdorff topological group $G$ and a closed subgroup $H$ of $G$ such that $d(H)>d(G)=\alpha$ (here $d$ denotes "density character"). Specifically, for $G$ we take the (appropriately topologized) free Abelian group $A(\beta D)$ generated by the Stone-Cech compactification of the discrete space $D$ with $|D|=\alpha$.
\end{abstract}

The principal result. For a space $X$, the free Abelian group $A(X)$ and its topology are defined as usual (see Markov [M] or Graev [G]; or see [HR, Theorem 8.8] for a detailed exposition of the non-Abelian analogue). In particular we have $A(X) \subset P=\prod_{i \in I} G_{i}$, where $\left\{\left(G_{i}, f_{i}\right): i \in I\right\}$ is an enumeration of all topological Abelian groups $G_{i}$ and continuous functions $f_{i}$ from $X$ to $G_{i}$ (with, say, $G_{i} \subset P P(X)$, this latter condition ensuring that $I$ is a set). We need the following basic properties.

(i) $X$ is a closed subspace of $A(X)$;

(ii) algebraically, $A(X)$ is the free Abelian group generated by $X$;

(iii) for every continuous function $f$ from $X$ into a (Hausdorff) Abelian group $G$ there is a continuous homomorphism $\bar{f}: A(X) \rightarrow G$ such that $f \subset \bar{f}$.

In what follows we fix the infinite cardinal $\alpha ; D$ and $\beta D$ are as defined in the abstract. We set $G=A(\beta D)$ and we denote by $H$ the subgroup of $G$ generated (in the group-theoretic sense) by the set $U(\alpha)$ of uniform ultrafilters on $\alpha$; these are by definition the ultrafilters $p \in \beta D$ such that $A \in p$ implies $|A|=\alpha$. We use additive notation, so that

$$
G=\left\{\sum_{k=1}^{n} z_{k} \cdot p_{k}: n<\omega, z_{k} \in Z, p_{k} \in \beta D\right\} .
$$

LEMma $1 . d(G)=\alpha$.

Proof. For $n<\omega$, define

$$
G_{n}=(\beta D)^{n}=\left\{\sum_{k=1}^{n} z_{k} \cdot p_{k}: z_{k} \in Z, p_{k} \in \beta D\right\} .
$$

Received by the editors July 9, 1975.

AMS (MOS) subject classifications (1970). Primary 22D05, 54A25; Secondary 22A05.

Key words and phrases. Topological group, free topological group, density character, closed subgroup. 
Then $G=\cup_{n<\omega} G_{n}$ and $d\left(G_{n}\right)=\alpha$, so that $d(G) \leqslant \omega \cdot \alpha=\alpha$.

Let $S \subset G$ be a subset of cardinality $<\alpha$. Let $F \subset \beta D$ be the set of all elements occuring in a minimal representation of some element of $S$ as integral combination of members of $\beta D$. Then $|F|<\alpha$. Then $\bar{F} \neq \beta D$. Let $x_{0}$ $\in \beta D-\bar{F}$. Then $\exists f: \beta D \rightarrow[0,1] \ni f$ is continuous and $f\left(x_{0}\right)=1$ and $f(\bar{F})=\{0\}$. Let $\tilde{f}$ be its continuous homomorphic extension from $G \rightarrow \pi$. Then $\tilde{f}(S)=\{0\}$ and $\tilde{f}\left(x_{0}\right)=1$. So $\bar{S} \neq G$. So $d(G) \geqslant \alpha$.

LEMMA 2. $H$ is a closed subgroup of $G$.

Proof. Let $x=\sum_{k=1}^{n} z_{k} \cdot p_{k} \in G \backslash H$; we assume without loss of generality that $p_{1} \notin U(\alpha), z_{1} \neq 0$, and $p_{k} \neq p_{1}$ for $2 \leqslant k \leqslant n$. There is a continuous function $f$ from $\beta D$ to the group of real numbers such that $f\left(p_{1}\right)=1$ and $f\left(p_{k}\right)=0$ for $2 \leqslant k \leqslant n$ and $f(p)=0$ for all $p \in U(\alpha)$. Clearly $\bar{f}(p)=0$ for all $p \in H$, while $\bar{f}(x)=z_{1} \neq 0$. Thus $x \notin \mathrm{cl}_{G} H$.

LEMMA 3. $d(H)>\alpha$.

Proof. Suppose that $E=\{x(\xi): \xi<\gamma\}$ is dense in $H$ with $\omega \leqslant \gamma \leqslant \alpha$, say with

$$
x(\xi)=\sum_{k=1}^{n(\xi)} z_{k}(\xi) \cdot p_{k}(\xi) \quad\left(n(\xi)<\omega, z_{k}(\xi) \in Z, p_{k}(\xi) \in U(\alpha)\right) .
$$

We define $S=\left\{p_{k}(\xi): \xi<\gamma, k \leqslant n(\xi)\right\}$, so that $|S| \leqslant \omega \cdot \gamma=\gamma$. It is well known (see for example [GJ, Exercise 12B], or [CN, Corollary 12.20]) that the space $U(\alpha)$ admits a family $Q$ of nonempty, pairwise disjoint, open subsets such that $|Q|>\alpha$. It follows that $S$ is not dense in $U(\alpha)$; we choose $p \in U(\alpha) \backslash \mathrm{cl}_{U(\alpha)} S$. As in the proof of Lemma 2 there is a continuous, realvalued function $f$ on $U(\alpha)$ such that $f(p)=1$ and $f(q)=0$ for all $q$ $\in \operatorname{cl}_{U(\alpha)} S$. Since $U(\alpha)$ is closed in the normal space $\beta D$, the function $f$ extends continuously to a real-valued function $g$ defined on $\beta D$ and we have $\bar{g}(p)=g(p)=1$ and $\bar{g}(q)=0$ for all $q \in S$; since $\bar{g}$ is a homeomorphism, it follows that $\bar{g}(q)=0$ for all $q \in E$. Since $E$ is dense in $H$ and $p \in H$ we have a contradiction. So the proof is complete.

Finally, the theorem announced in the abstract follows immediately from Lemmas 1,2 and 3.

REMARK. It is known (see [CIR] and [TSR]) that if $H$ is a closed subgroup of a locally compact group $G$, then $d(H) \leqslant d(G)$.

ACKNowledgement. Thanks are due to Professor W. W. Comfort for his kind encouragment and interest that helped this paper to be in this form.

\section{REFERENCES}

[CIR] W. W. Comfort, Gerald L. Itzkowitz and Kenneth A. Ross, Density character in topological groups (to appear).

[CN] W. W. Comfort and S. Negrepontis, The theory of ultrafilters, Grundlehren der math. Wissenschaften, vol. 211, Springer-Verlag, Heidelberg, 1974.

[G] M. I. Graev, Free topological groups, Izv. Akad. Nauk SSSR Ser. Mat. 12 (1948), 279-324; English transl., Amer. Math. Soc. Transl. (1) 8 (1962), 305-364. MR 10, 11.

[GJ] Leonard Gillman and Meyer Jerison, Rings of continuous functions, University Ser. in Higher Math., Van Nostrand, Princeton, N.J., 1960. MR 22 \#6994. 
[HR] Edwin Hewitt and Kenneth A. Ross, Abstract harmonic analysis. Vol. I. Integration theory, group representations, Die Grundlehren der math. Wissenschaften, Band 115, Academic Press, New York; Springer-Verlag, Berlin, 1963. MR 28 \# 158.

[M] A. A. Markov, On free topological groups, Izv. Akad. Nauk SSSR Ser. Mat. 9 (1945), 3-64; English transl., Amer. Math. Soc. Transl. (1) 8 (1962), 195-272. MR 7, 7.

[TSR] T. Soundararajan, Weakly Hausdorff spaces and the cardinality of topological spaces, General Topology and its Relations to Modern Analysis and Algebra, III (Proc. Conf., Kanpur, 1968), Academia, Prague, 1971, pp. 301-306. MR 44 \#976.

Depattment of Mathematics, University of Manitoba, Winnipeg, Manitoba, Canada

Department of Mathematics, Memphis State University, Memphis, Tennessee 38152

Department of Mathematics, University of Costa Rica, San Jose, Costa Rica 